\title{
Calcium-binding and coiled-coil domain 2 promotes the proliferation and suppresses apoptosis of prostate cancer cells
}

\author{
FEILUN CUI $^{1}$, SIJIA WANG ${ }^{2}$, JIAN TAN $^{1}$, HUAMING TANG $^{1}$, YU FAN $^{3}$ and JIANPENG HU ${ }^{1}$ \\ ${ }^{1}$ Department of Urology, The Affiliated People's Hospital, Jiangsu University, Zhenjiang, Jiangsu 212012; \\ ${ }^{2}$ Department of Basic Medicine, Air Force Medical University, Xi'an, Shaanxi 710032; ${ }^{3}$ Department of Oncology, \\ The Affiliated People's Hospital, Jiangsu University, Zhenjiang, Jiangsu 212012, P.R. China
}

Received September 14, 2018; Accepted September 11, 2020

DOI: $10.3892 /$ etm.2021.9836

\begin{abstract}
Prostate cancer (PCa) is considered to be one of the most common tumors in men. Calcium-binding and coiled-coil domain 2 (CALCOCO2) is a known important xenophagy receptor, which mediates intracellular bacterial degradation. To the best of the authors' knowledge, the present study is the first to demonstrate that CALCOCO2 functions as an oncogene in PCa. The results of the current study indicated that CALCOCO2 knockdown suppressed cell proliferation and colony formation, whereas it promoted apoptosis of PCa cells. In addition, knockdown of CALCOCO2 in PCa cells reduced cyclin-E1 and increased $\mathrm{p} 53$ protein expression. Bioinformatics analysis revealed that $\mathrm{CALCOCO} 2$ was associated with 'autophagosome assembly', 'nucleophagy' and 'nucleic acid metabolic process' biological processes and interacted with sequestosome-1, microtubule-associated proteins $1 \mathrm{~A} / 1 \mathrm{~B}$ light chain 3 (MAP1LC3)B, $\gamma$-aminobutyric acid receptorassociated protein, IкB kinase subunit $\gamma$ and MAP1LC3C. Moreover, $\mathrm{CALCOCO} 2$ protein levels were indicated to be significantly increased in PCa samples compared with normal prostate tissues. These results suggested that $\mathrm{CALCOCO} 2$ may be of value as a diagnostic and therapeutic target in PCa.
\end{abstract}

\section{Introduction}

Autophagy, which is a highly conserved evolutionary process, degrades harmful protein aggregates and intracellular toxins to provide nutrients, such as amino acids, nucleotides and

Correspondence to: Professor Jianpeng $\mathrm{Hu}$, Department of Urology, The Affiliated People's Hospital, Jiangsu University, 8 Dianli Road, Zhenjiang, Jiangsu 212012, P.R. China

E-mail: 249056614@qq.com

Professor Yu Fan, Department of Oncology, The Affiliated People's Hospital, Jiangsu University, 8 Dianli Road, Zhenjiang, Jiangsu 212012, P.R. China

E-mail: yuf36@sina.com

Key words: prostate cancer, calcium-binding and coiled-coil domain 2, proliferation, apoptosis fatty acids, that aid in sustaining ATP production in cells $(1,2)$. Prostate cancer ( $\mathrm{PCa}$ ) has been revealed to be one of the most common tumors in men worldwide (3). Emerging evidence has indicated that autophagy may promote tumor progression and resistance to treatment in advanced PCa (4-6). Multiple autophagy regulators, such as sequestosome-1 (SQSTM1/p62) (7,8) and autophagy related 7 (ATG7) (9), have been demonstrated to serve key roles in PCa progression. For example, increased ATG7 expression and decreased PTEN levels have been revealed to drive PCa growth (9). Xenophagy is a type of selective autophagy that is associated with the removal of intracellular pathogens $(10,11)$. Of note, xenophagy may also affect tumor progression and treatment (12-14). For example, Helicobacter pylori- or Epstein-Barr virus-induced xenophagy has been associated with gastric tumorigenesis (14). However, the role of xenophagy in PCa remains largely elusive.

Calcium-binding and coiled-coil domain 2 (CALCOCO2; also referred to as NDP52) is considered to be the most important known xenophagy receptor (15). Xenophagy has been indicated to protect the mammalian cytosol against bacterial infection $(15,16)$. CALCOCO2 has been revealed to interact with microtubule-associated proteins 1A/1B light chain 3 (MAP1LC3)A, MAP1LC3B and/or $\gamma$-aminobutyric acid receptor-associated protein (GABARAP)-like (GABARAPL) 2 to regulate bacterial targeting via autophagosomes, and with MAP3LC3C to regulate pathogen-containing autophagosome maturation $(17,18)$. Moreover, a previous study has demonstrated that CALCOCO2 binding to galectin-8 activated antibacterial autophagy to suppress the proliferation of infecting pathogens (19). CALCOCO2 may also bind to influenza virus protein PB1-F2 to modulate the innate immune response (20). Of note, a number of studies have indicated that CALCOCO2 may be associated with human cancer progression by interacting with tumor-related pathways, such as ІкB kinase and the NF- $\kappa B$ pathway (20). Using a CALCOCO2 interacting protein dataset, which was downloaded from The National Center for Biotechnology Information, the present study revealed that $\mathrm{CALCOCO} 2$ was associated with 'autophagosome assembly', 'nucleophagy' and 'nucleic acid metabolic process'. Exploring the roles of CALCOCO2 in $\mathrm{PCa}$ may provide a potential biomarker for cancer treatment.

The purpose of the present study was to explore the potential roles of CALCOCO2 in PCa. Loss-of-function assays were 
performed to detect the effect of CALCOCO2 on PCa cell proliferation, apoptosis and colony-forming ability. Moreover, bioinformatics analysis was performed to identify the mechanisms of function of CALCOCO2 in PCa. The results of the current study demonstrated that CALCOCO2 may be of value as a novel therapeutic and prognostic target for $\mathrm{PCa}$.

\section{Materials and methods}

Cell culture. 293T, PC-3 and DU145 cells were purchased from The Cell Bank of Type Culture Collection of the Chinese Academy of Sciences (Shanghai, China). The cells were verified using DNA-fingerprinting and mycoplasma, isozyme and cell vitality detection (data not shown). The cells were maintained in RPMI-1640 medium supplemented with $10 \%$ FBS (both from Gibco; Thermo Fisher Scientific, Inc.) and $1 \%$ penicillin/streptomycin (Thermo Fisher Scientific, Inc.). Cultures were maintained at $37^{\circ} \mathrm{C}$ in a $5 \% \mathrm{CO}_{2}$ cell incubator.

Lentiviral constructs and infection. Human lentivirus-short hairpin (sh)CALCOCO2 and lentivirus-shControl (shCtrl) sequences were designed and purchased from Shanghai GeneChem Co., Ltd. Recombinant lentiviral vectors carrying CALCOCO2 shRNA were constructed using standard molecular techniques according to our previous study (21). Briefly, 293T cells were infected with 1,200 ng pCV146-Luc-Puromycin-shCALCOCO2 or shCtrl vector, 900 ng pHelper 1.0 vector (Shanghai GeneChem Co., Ltd.) and $600 \mathrm{ng}$ pHelper 2.0 vector (Shanghai GeneChem Co., Ltd.) using $7.5 \mu 1$ Lipofectamine ${ }^{\circledR} 2000$ (Thermo Fisher Scientific, Inc.) to generate stably transfected cells. A total of $4 \mathrm{~h}$ after transfection, Opti-modified Eagle's medium (Opti-MEM; cat. no. 31985062; Thermo Fisher Scientific, Inc.) was changed to RPMI-1640 medium containing 10\% FBS (Cytiva) and were cultured at $37^{\circ} \mathrm{C}$ in $5 \% \mathrm{CO}_{2}$. After $24 \mathrm{~h}$, concentrated lentiviruses were collected. Concentrated lentiviruses were transfected into DU145 and PC-3 cells at a MOI of 40 in serum-free RPMI-1640 medium. The supernatant was replaced with complete culture medium (RPMI-1640 medium containing 10\% FBS) after $24 \mathrm{~h}$. Following $48 \mathrm{~h}$ the infection efficiency of CALCOCO2 shRNA was detected via reverse transcription-quantitative PCR (RT-qPCR) analysis. The CALCOCO2 shRNA sequence was 3'-CCGGGAGCTGCTTCAACTGAAAGAACTCGA GTTCTTTCAGTTGAAGCAGCTCTTTTT-5', and the shCtrl sequence was 5'-CCGGTCTTCTCCGAACGTGTCACGTCT CGAGACGTGACACGTTCGGAGAAGATTTTTG-3'.

$R T$-qPCR. TRIzol ${ }^{\circledR}$ reagent (Invitrogen; Thermo Fisher Scientific, Inc.) was used to extract total RNA from PCa cell lines. RNA $(1 \mu \mathrm{g})$ was reverse transcribed into complementary DNA according to the protocol of PrimeScript RT reagent kit (Takara Biotechnology Co., Ltd.). The reaction conditions for the RT were as follows: $25^{\circ} \mathrm{C}$ for $5 \mathrm{~min}, 50^{\circ} \mathrm{C}$ for $25 \mathrm{~min}$ and $75^{\circ} \mathrm{C}$ for 10 min. qPCR was performed using the iQ SYBR ${ }^{\circledR}$-Green Supermix and ABI Prism 7900 platform (both from Bio-Rad Laboratories, Inc.), according to the manufacturer's protocol. The PCR cycling conditions were as follows: $50^{\circ} \mathrm{C}$ for $2 \mathrm{~min}$, followed by $95^{\circ} \mathrm{C}$ for $10 \mathrm{~min} ; 40$ cycles of $95^{\circ} \mathrm{C}$ for $15 \mathrm{sec}$; and $60^{\circ} \mathrm{C}$ for $1 \mathrm{~min}$. GAPDH was used as an internal control. The $2^{-\Delta \Delta \mathrm{Cq}}$ method was used to calculate the relative expression level and GAPDH was used as the internal reference gene (22). The primers used were as follows: $\mathrm{CALCOCO} 2$ forward, 5'-TGAAGGAGGCGCAAGACAAAA-3' and reverse, 5'-CAT CTGCTGTTGCTCCAAGGT-3'; GAPDH forward, 5'-TGA CTTCAACAGCGACACCCA-3' and reverse, 5'-CACCCT GTTGCTGTAGCCAAA-3'. All primers were synthesized by Shanghai GeneChem Co., Ltd. Each sample was run in triplicate to ensure quantitative accuracy.

Western blotting. Following transfection for 48 h, DU145 and PC-3 cell lysates were prepared using RIPA lysis buffer and protease cocktail inhibitor I (Merck KGaA). After quantification with a BCA assay kit (Thermo Fisher Scientific, Inc.), $10 \mu \mathrm{g}$ proteins per lane were separated using $10 \%$ SDS-PAGE and subsequently transferred to a PVDF membrane. After blocking in 5\% non-fat milk at room temperature for $1 \mathrm{~h}$, the membranes were washed with TBS $+0.1 \%$ Tween- 20 . Membranes were incubated with primary antibodies against cyclin-E1 (1:1,000; cat. no. 4129; Cell Signaling Technology, Inc.); 53 (1:1,000; cat. no. 2527; Cell Signaling Technology, Inc.), GAPDH (1:1,000; cat. no. sc-32233; Santa Cruz Biotechnology, Inc.) at $4^{\circ} \mathrm{C}$ overnight, followed by incubation with HRP-conjugated anti-rabbit IgG (1:2,000; cat. no. 7074) or HRP-conjugated anti-mouse IgG (1:2,000; cat. no. 7076; both from Cell Signaling Technology, Inc.) secondary antibodies for $1 \mathrm{~h}$ at room temperature. Protein bands were visualized using Pierce ECL Western Blotting Substrate (Thermo Fisher Scientific, Inc.). The protein band intensity was analyzed using ImageJ software, v1.41 (National Institutes of Health).

Plate analysis using the Celigo adherent cell cytometry system. The Celigo adherent cell cytometry system (Nexcelom Bioscience LLC) was used for rapid quantification of cellular fluorescence expression, as previously described (23). Following CALCOCO 2 knockdown, PC-3 and DU145 cells were seeded into 96-well plates $\left(1.5 \times 10^{3}\right.$ cells/well) and cultured at $37^{\circ} \mathrm{C}$ in a $5 \% \mathrm{CO}_{2}$ cell incubator for 5 days. Cell counting was performed using the Celigo Imaging Cytometer and the captured cell images were analyzed using Celigo software version 1.0 (both from Nexcelom Bioscience LLC).

Cell apoptosis assay. A total of $1 \times 10^{5}$ shCALCOCO2 and shCtrl stably transfected PC-3 and DU145 cells were harvested and washed with PBS three times. Cells were assayed with an Annexin V-APC Apoptosis Detection kit (cat. no. 88-8007-74; eBioscience; Thermo Fisher Scientific, Inc.) and were analyzed using a flow cytometer (BD FASCCalibur; BD Biosciences). Furthermore, Annexin V-FITC/PI staining was also performed using flow cytometry (cat. no. 88-8005-72; eBioscience; Thermo Fisher Scientific, Inc.). Briefly, $1 \times 10^{5}$ shCALCOCO2 and shCtrl PC-3 and DU145 cells were collected and washed with ice-cold PBS. Subsequently, the cells were resuspended in $300 \mu 1$ binding buffer, which was included in the Annexin V-FITC/PI kit. The cells were incubated with $5 \mu \mathrm{l}$ Annexin V-FITC and $5 \mu \mathrm{l}$ propidium iodide (PI) in the dark for $15 \mathrm{~min}$ at room temperature. Finally, the cells were analyzed via flow cytometry (BD FASCCalibur; 
BD Biosciences). The total percentage of apoptotic cells was defined as the sum of both early apoptosis (Annexin V-FITC positive, PI negative) and late apoptosis (Annexin V-FITC PI positive), top and bottom right quadrants in flow cytometric dot plots, respectively.

Colony formation assays. shCALCOCO2 and shCtrl stably transfected PC-3 and DU145 cells were seeded into six-well plates with a density of 500 cells/well. After 10 days in a $37^{\circ} \mathrm{C}$ incubator, cell colonies (>50 cells) were counted via staining with $0.5 \%$ crystal violet solution (Sigma-Aldrich; Merck $\mathrm{KGaA}$ ) for $30 \mathrm{sec}$ at room temperature following fixation with $10 \%$ formaldehyde for $5 \mathrm{~min}$ at room temperature. The stained colonies were observed under a light microscope at a magnification of $\mathrm{x} 20$ (Nikon Corporation) and counted using ImageJ software (version 4.0; National Institutes of Health). Triplicate wells were measured for each treatment group.

Gene Ontology (GO) and pathway enrichment analysis of differentially expressed genes. GO analysis and Kyoto Encyclopedia of Genes and Genomes pathway analysis (24) were performed. The Database for Annotation, Visualization and Integrated Discovery (david.abcc.ncifcrf.gov), was used to integrate functional genomic annotations (25). $\mathrm{P}<0.05$ was considered to indicate a statistically significant difference.

Integration of the protein-protein interaction (PPI) network. The Search Tool for the Retrieval of Interacting Genes/Proteins version 10.0 (STRING; string-db.org) was used for the exploration of potential interactions among genes (26). A PPI score of $>0.4$ was considered significant. The PPI networks were visualized using Cytoscape software version 3.6.1 (http://www. cytoscape.org) (27). $\mathrm{P}<0.05$ was considered to indicate a statistically significant difference.

Public datasets analysis. The present study analyzed CALCOCO 2 protein levels in PCa tissues using a public dataset, which was derived from The Human Protein Atlas (HPA; https://www.proteinatlas.org/) (28). HPA, as a database containing images from immunohistochemical-based tissue microarrays (46 normal human tissues and 20 types of human cancer) for 11,250 human proteins, was accessed to analyze CALCOCO2proteinexpressioninPCatissuesandnon-cancerous prostate tissues, and the images and expression levels of CALCOCO2 were downloaded from the website following guidelines (https://www.proteinatlas.org/ENSG00000136436CALCOCO2/pathology/prostate+cancer). To examine the effect of CALCOCO2 on PCa cell survival, the gene-level differential dependency scores (https://depmap.org/rnai/index) were investigated to assess the association of CALCOCO2 with tumor survival (29). The CALCOCO2 interacting protein map was directly downloaded from NCBI (https://www.ncbi. nlm.nih.gov/gene/10241).

Statistical analysis. The differences between two groups in terms of gene expression, cell apoptosis, colony number and migrating cell number were evaluated using unpaired Student's t-test. All statistical analyses were performed using GraphPad Prism software v5.0 (GraphPad Software, Inc.).
$\mathrm{P}<0.05$ was considered to indicate a statistically significant difference.

\section{Results}

CALCOCO2 knockdown inhibits the proliferation of PCa cells. DepMap database analysis indicated that CALCOCO 2 knockdown suppressed the proliferation of androgen receptor-dependent LNCaP cells (Fig. S1). In order to evaluate the effect of CALCOCO2 on PCa progression, specific shRNAs were designed to target CALCOCO2 in the PCa cell lines PC-3 and DU145. As presented in Fig. 1A and B, PC-3 and DU145 cells transfected with shCALCOCO2 lentiviral plasmid exhibited significantly lower CALCOCO2 expression levels compared with those transfected with shCtrl lentiviral plasmid.

Subsequently, the role of CALCOCO2 in the regulation of PCa cell proliferation was examined using the Celigo Cell Counting assay. Cell counting revealed that the PCa cell proliferation rate was significantly lower in shCALCOCO2 PC-3 (Fig. 1C and E) and DU145 (Fig. 1D and F) cells compared with the shCtrl groups.

Knockdown of CALCOCO2 suppresses PCa cell colony formation. The effects of CALCOCO2 knockdown on DU145 and PC-3 cell tumorigenesis potential were examined in vitro by assessing colony formation. As indicated in Fig. 2, knockdown of CALCOCO2 resulted in significant alterations in the ability of PCa cells to form colonies. Compared with a mean of 100 colonies in shCtrl DU145 and PC-3 cells, CALCOCO2 knockdown significantly suppressed PCa cell colony formation to a mean of 28 colonies in DU145 cells (Fig. 2A and B) and 3 colonies in PC-3 cells (Fig. 2C and D).

Knockdown of CALCOCO2 promotes apoptosis of PCa cells. The present study examined the apoptosis of PCa cells using Annexin V staining following CALCOCO2 knockdown. As demonstrated in Fig. 3, the percentage of apoptotic cells in the shCALCOCO2 group was significantly increased compared with shCtrl cells both in the DU145 (Fig. 3A and B) and PC-3 (Fig. 3C and D) cell lines.

In order to further investigate the apoptosis-inducing effects of CALCOCO2 on PCa cells, DU145 and PC-3 cells following CALCOCO2 knockdown were stained with Annexin V and PI, and subsequently analyzed using flow cytometry. The early and late apoptotic rates of shCALCOCO2 DU145 (Fig. 4A and B) and PC-3 (Fig. 4C and D) cells were significantly increased compared with the control groups. The combined quantification of the early and late apoptotic rates is presented in Fig. 4B and D.

Knockdown of CALCOCO2 suppresses cyclin-E1 and promotes p53 expression in PCa cells. In order to elucidate the potential mechanisms of action of CALCOCO2 in the regulation of $\mathrm{PCa}$ cell proliferation and apoptosis, the effects of CALCOCO2 knockdown on cyclin-E1 and p53 expression, which are considered to be key regulators of cancer growth $(30,31)$, were detected. The results demonstrated that knockdown of CALCOCO2 suppressed cyclin-E1 protein levels in both DU145 (Fig. 5A and B) 

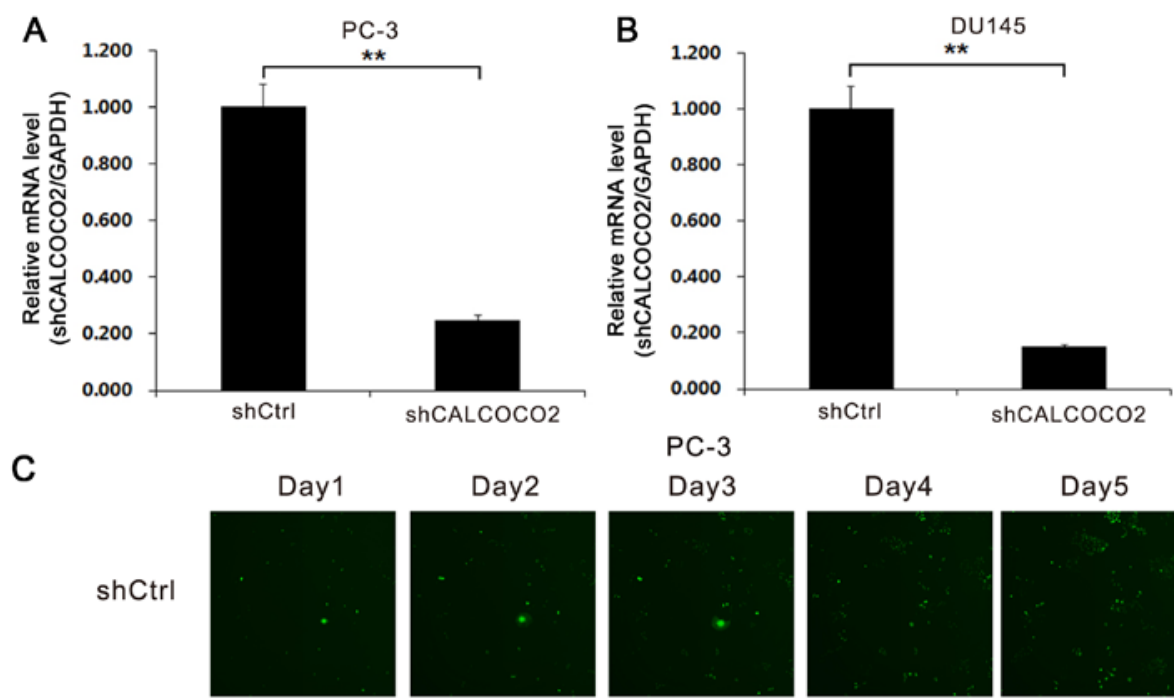

Day2

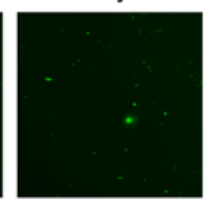

$\mathrm{PC}-3$
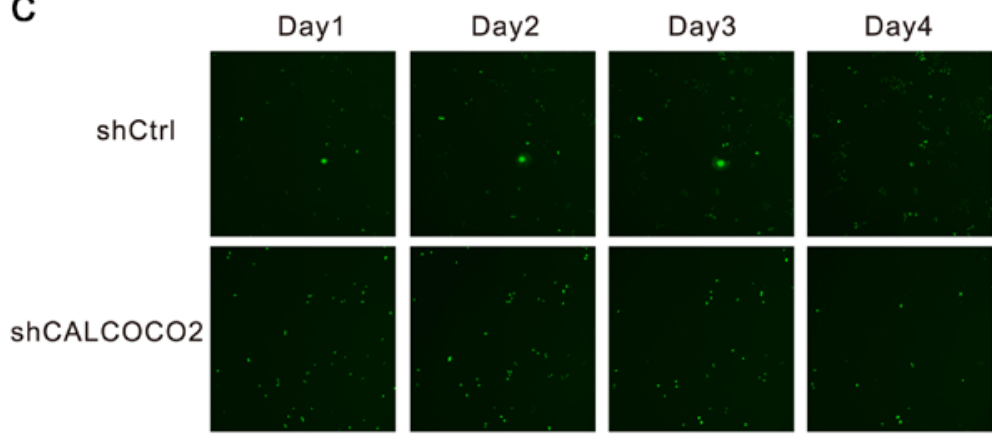

Day5

D

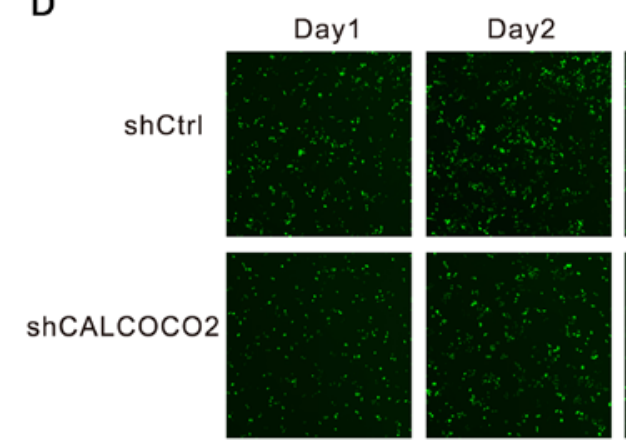

Day2
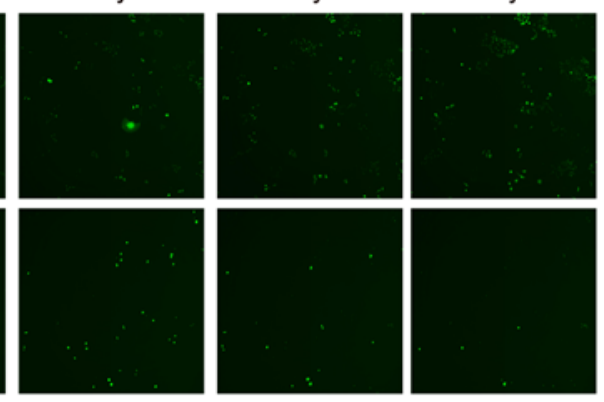

DU145
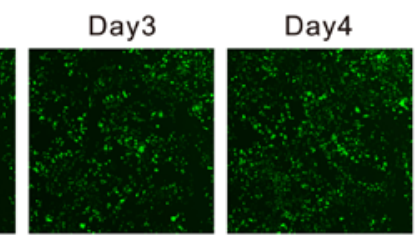

Day5

E
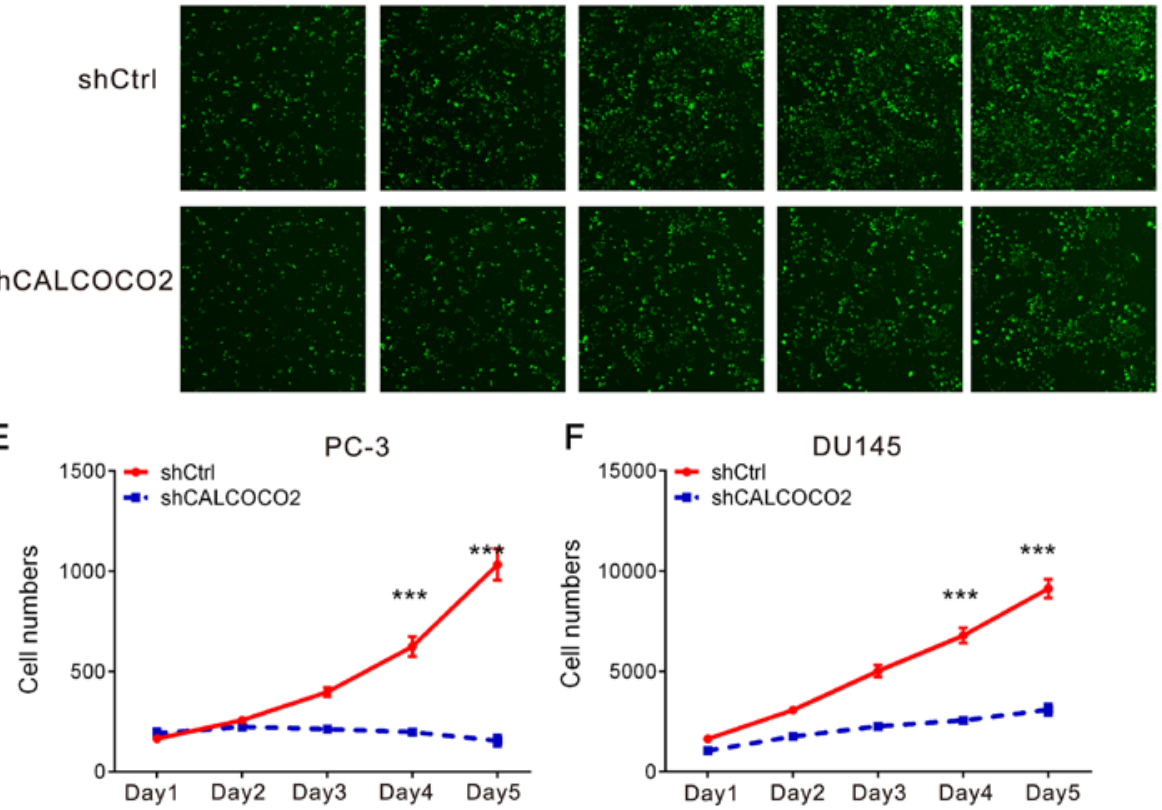

F

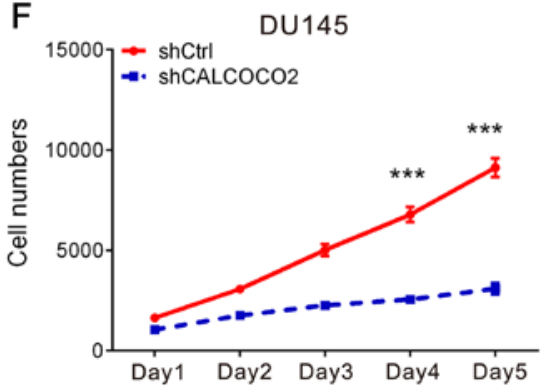

Figure 1. CALCOCO2 knockdown inhibits proliferation of prostate cancer cells. Reverse transcription-quantitative PCR analysis indicated that CALCOCO2 mRNA level was reduced following CALCOCO2 knockdown in (A) PC-3 and (B) DU145 cells. Detection of proliferation using the Celigo ${ }^{\circledR}$ system revealed that knockdown of CALCOCO2 inhibited the proliferation of (C) PC-3 cells and (D) DU145 cells (magnification, x100). Quantification of cell numbers indicated a significant decrease in the shCALCOCO2 group compared with the shCtrl group of (E) PC-3 and (F) DU145 cells. The results are presented as the mean \pm standard deviation $(n=3) .{ }^{* *} \mathrm{P}<0.01$ and ${ }^{* * *} \mathrm{P}<0.001$ vs. shCtrl. CALCOCO2, calcium binding and coiled-coil domain 2; sh, short hairpin; Ctrl, control.

and PC-3 (Fig. 5C and D) cells. However, silencing of CALCOCO2 increased p53 protein levels in DU145 cells (Fig. 5E and F).

Bioinformatics analysis of CALCOCO2 in PCa. In the present study, CALCOCO2-mediated PPI networks were constructed to predict its potential roles in PCa progression. A total of 190 proteins, including GABARAPL1, MAP1LC3B, GABARAP, IкB kinase subunit $\gamma$, MAP1LC3C, SQSTM1 and I $\mathrm{KB}$ kinase epsilon, were identified as CALCOCO2 cofactors using a CALCOCO2 interacting proteins dataset, which was downloaded from NCBI (https://www.ncbi.nlm. nih.gov/gene/10241). Subsequently, CALCOCO2-mediated PPI networks were constructed using the STRING database. A total of 97 nodes and 310 edges were included in the CALCOCO2-associated PPI network (Fig. 6A).

$\mathrm{GO}$ analysis was also performed to predict the potential roles of CALCOCO2 using STRING database. GO analysis revealed that CALCOCO2 was primarily associated with 'mitophagy', 'macroautophagy', 'nucleophagy', 'nucleic acid 
A

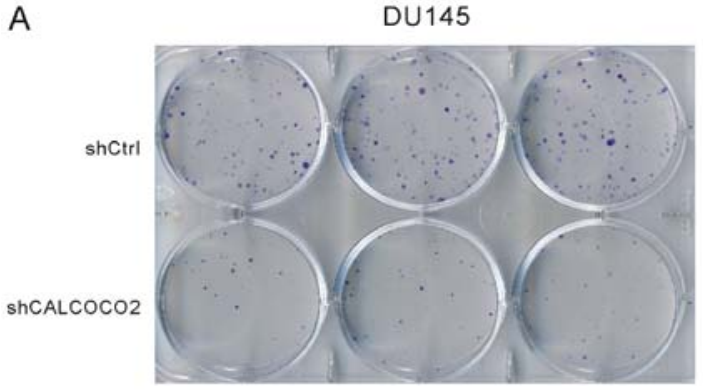

C

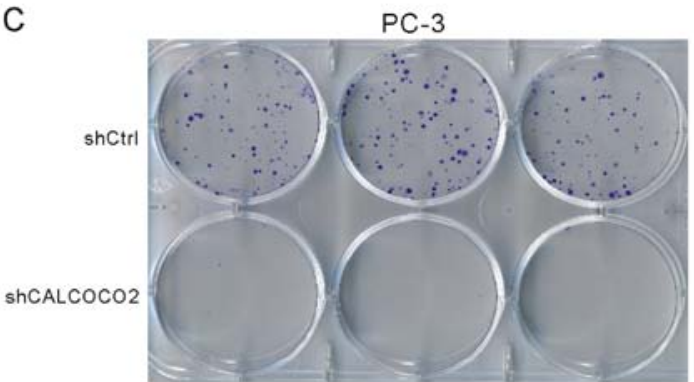

B

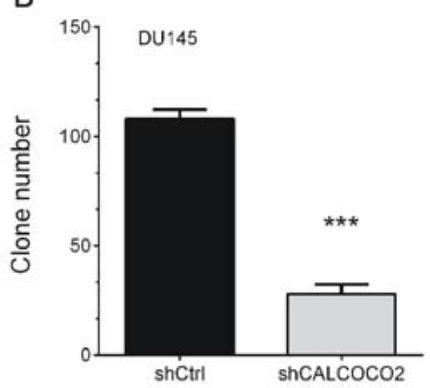

D

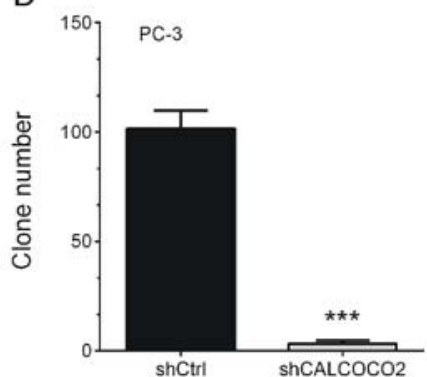

Figure 2. CALCOCO2 knockdown reduces colony formation of prostate cancer cells. CALCOCO2 knockdown significantly reduced colony formation of (A and B) DU145 and (C and D) PC-3 cells (magnification, $\mathrm{x} 20)$. The results are presented as the mean \pm standard deviation $(\mathrm{n}=3) .{ }^{* * * *} \mathrm{P}<0.001 \mathrm{vs}$. shCtrl. CALCOCO2, calcium binding and coiled-coil domain 2; sh, short hairpin; Ctrl, control.

A
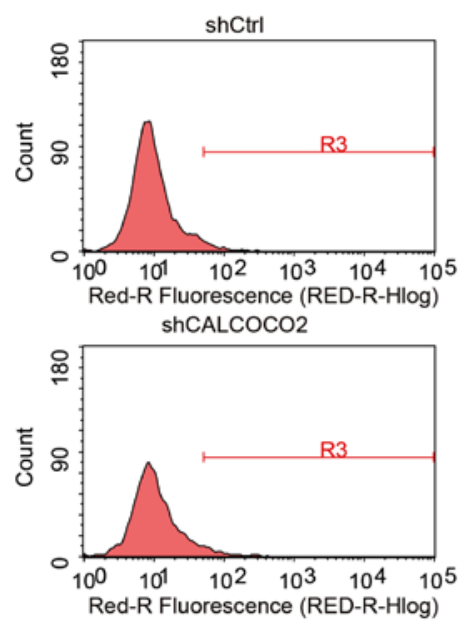

$\mathrm{C}$
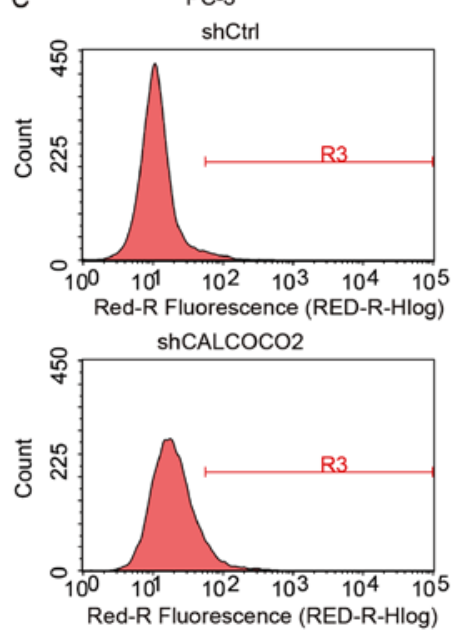

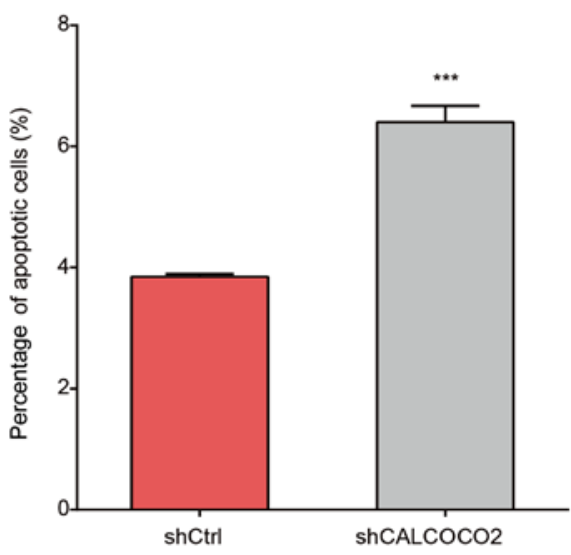

Cell apoptosis in DU145

$\mathrm{D}$

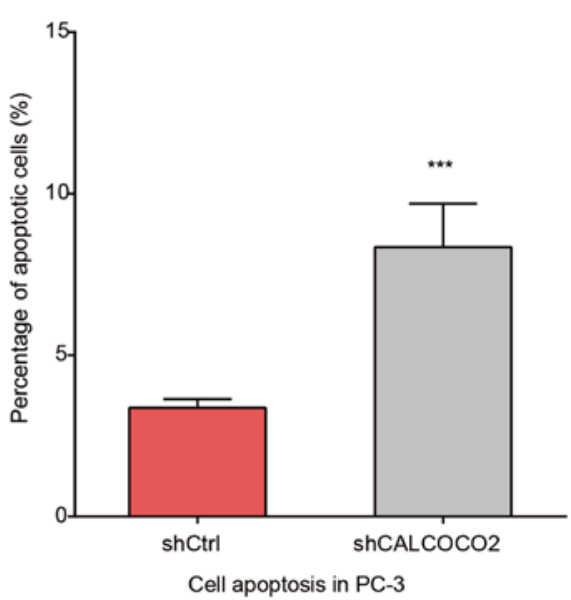

Figure 3. CALCOCO2 knockdown promotes apoptosis in prostate cancer cells. Flow cytometric analysis using an Annexin V-APC Apoptosis Detection kit indicated that CALCOCO2 knockdown promoted apoptosis of (A and B) DU145 and (C and D) PC-3 cells. The results are presented as the mean \pm standard deviation $(n=3) .{ }^{* * *} \mathrm{P}<0.001$ vs. shCtrl. CALCOCO2, calcium binding and coiled-coil domain 2; sh, short hairpin; Ctrl, control. 

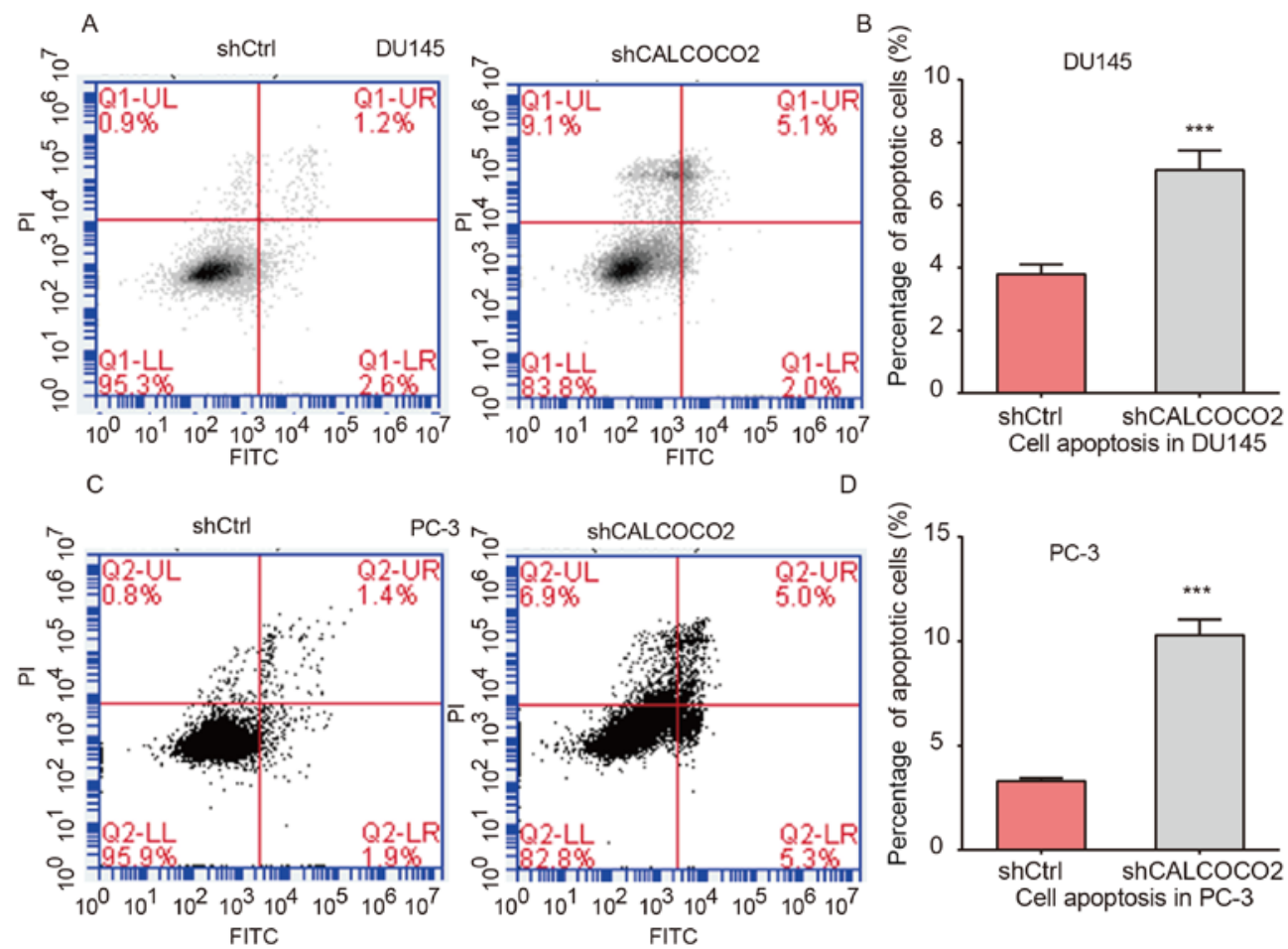

Figure 4. CALCOCO2 knockdown promotes early and late apoptosis in prostate cancer cells. Flow cytometric analysis using an Annexin V-FITC/PI Apoptosis Detection kits revealed that CALCOCO2 knockdown promoted early and late apoptosis of (A and B) DU145 and (C and D) PC-3 cells. The results are presented as the mean \pm standard deviation $(\mathrm{n}=3) .{ }^{* * *} \mathrm{P}<0.001$ vs. shCtrl. CALCOCO2, calcium binding and coiled-coil domain 2; sh, short hairpin; Ctrl, control; PI, propidium iodide.
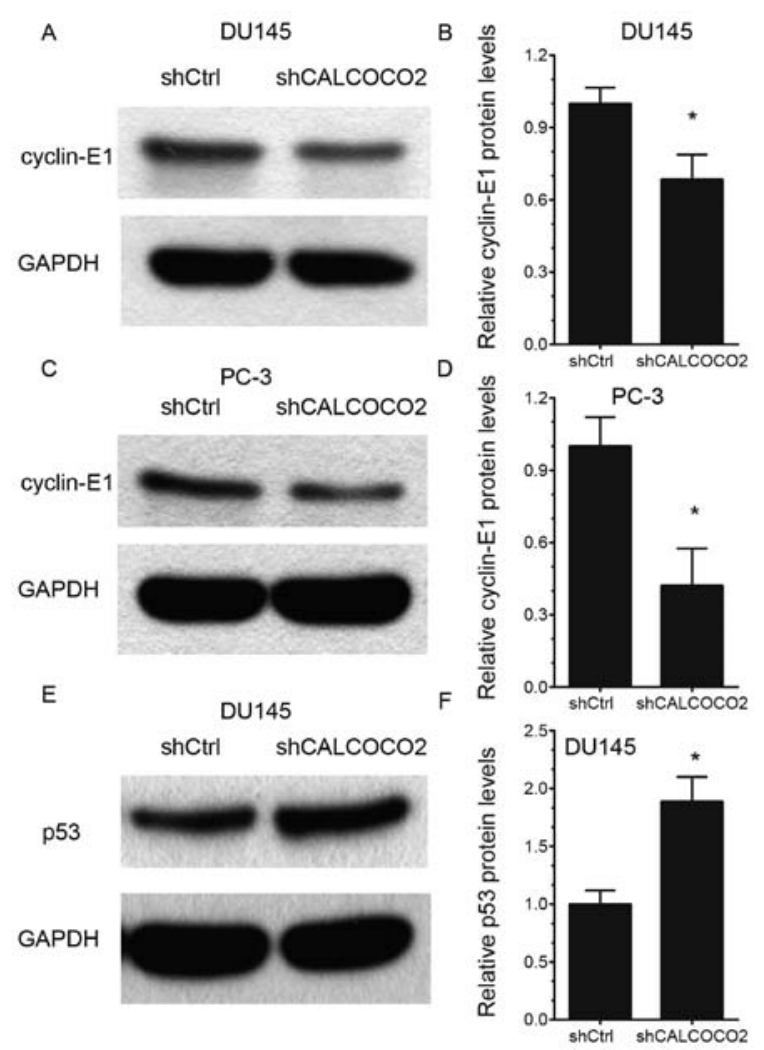

Figure 5. CALCOCO2 knockdown affects cyclin-E1 and p53 expression in prostate cancer cells. (A) Western blot assay and (B) quantification of cyclin-E1 expression levels following knockdown of CALCOCO2 in DU145 cells. (C) Western blot assay and (D) quantification of cyclin-E1 expression levels following knockdown of CALCOCO2 in PC-3 cells. (E) Western blot assay and (F) quantification of p53 expression levels following knockdown of CALCOCO2 in DU145 cells. " $\mathrm{P}<0.05$ vs. shCtrl. CALCOCO2, calcium binding and coiled-coil domain 2 ; sh, short hairpin; Ctrl, control. metabolic process', 'autophagosome assembly', 'regulation of transcription', 'regulation of gene expression', 'RNA metabolic process', 'nucleobase-containing compound metabolic process' and 'cytoplasmic pattern recognition receptor signaling pathway' (Fig. 6B). Molecular function analysis revealed that CALCOCO2 was associated with 'zinc ion binding', 'microtubule binding', 'transition metal ion binding', 'tubulin binding', 'protein binding', 'GABA receptor binding', 'nucleic acid binding' and 'tumor necrosis factor receptor binding' (Fig. 6C).

Protein levels of CALCOCO2 are increased in PCa samples. In order to additionally validate the oncogenic role of $\mathrm{CALCOCO} 2$ in $\mathrm{PCa}, \mathrm{CALCOCO} 2$ protein levels were analyzed in PCa tissues using a public dataset from The Human Protein Atlas. The results demonstrated that the protein levels of CALCOCO2 were not detectable in normal prostate tissues (Fig. 7A and B; https:// www.proteinatlas.org/ENSG00000136436-CALCOCO2/tissue/ prostate\#img). However, 9\% (1/11) of PCa samples exhibited high CALCOCO 2 protein levels, $18 \%$ (2/11) of PCa samples exhibited intermediate CALCOCO2 protein levels, 36\% (4/11) of PCa samples exhibited low CALCOCO2 protein levels (Fig. 7C and D, https://www.proteinatlas.org/ENSG00000136436-CALCOCO2/ pathology/tissue/prostate+cancer) and 36\% (4/11) of PCa samples exhibited no CALCOCO2 expression (data not shown). These results suggested that $\mathrm{CALCOCO} 2$ is overexpressed in $\mathrm{PCa}$ samples compared with normal tissues.

\section{Discussion}

$\mathrm{PCa}$ is considered to be one of the most common types of cancer affecting men worldwide (32). Autophagy has been indicated to serve an important role in cancer (33). A number of autophagy 
A

B

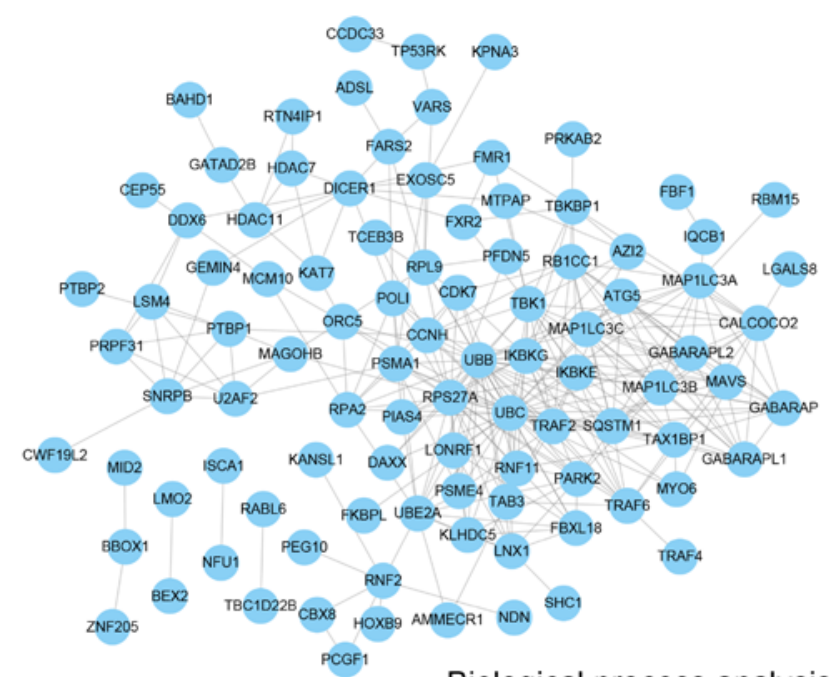

cytoplasmic pattern recognition receptor signaling pathway nucleobase-containing compound metabolic process

RNA metabolic process-

regulation of gene expression-

regulation of transcription-

autophagosome assembly-

nucleic acid metabolic process -

nucleophagy-

macroautophagy-

mitophagy-

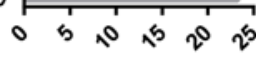

- $\log ($ P-value)

\section{C}

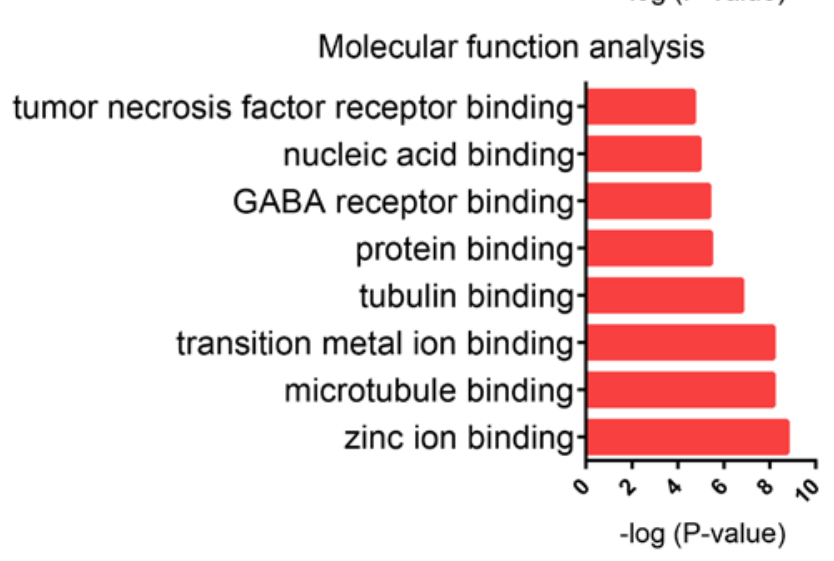

Figure 6. Bioinformatics analysis of CALCOCO2 in prostate cancer. (A) CALCOCO2-mediated PPI network. (B) Biological process analysis for CALCOCO2. (C) Molecular function analysis for CALCOCO2. CALCOCO2, calcium binding and coiled-coil domain 2; PPI, protein-protein interaction.

regulators have been associated with $\mathrm{PCa}$ progression. For example, beclin-1 has been revealed to be downregulated in PCa samples (34). Autophagy-associated protein p62 has been demonstrated to inhibit the autophagic flux and promote epithelial-to-mesenchymal transition (EMT) in metastatic PCa by sustaining histone deacetylase 6 expression (35). Moreover, ATG7 deficiency has been indicated to delay PTEN-deficient PCa progression (9). CALCOCO2 is considered to be the most important known xenophagy receptor; however, only few studies have focused on its potential role in human cancer (15). In the present study, CALCOCO2 expression was knocked down in PCa cell lines and the effect of this knockdown on the proliferation, colony formation and apoptosis of PCa cells was evaluated. The present study demonstrated that CALCOCO2 knockdown significantly inhibited cell proliferation and colony formation, whereas it promoted apoptosis of PCa cells. Apoptosis, which is a type of programmed cell death, has been indicated to serve an important role in cancer progression and evasion of apoptosis is considered to be a hallmark of cancer (36). Knockdown of CALCOCO2 in PCa cells reduced cyclin-E1 and increased p53 protein levels. Moreover, CALCOCO2 protein levels were indicated to be higher in PCa samples compared with normal prostate tissues in the analysis performed using The Human Protein Atlas. To the best of our knowledge, these results are the first to demonstrate that CALCOCO2 functions as an oncogene in PCa.

Previous studies have reported that $\mathrm{CALCOCO} 2$ can interact with a number of proteins, such as galectin-8 (19) and PB1-F2 (20), to mediate xenophagy. Several studies indicated 

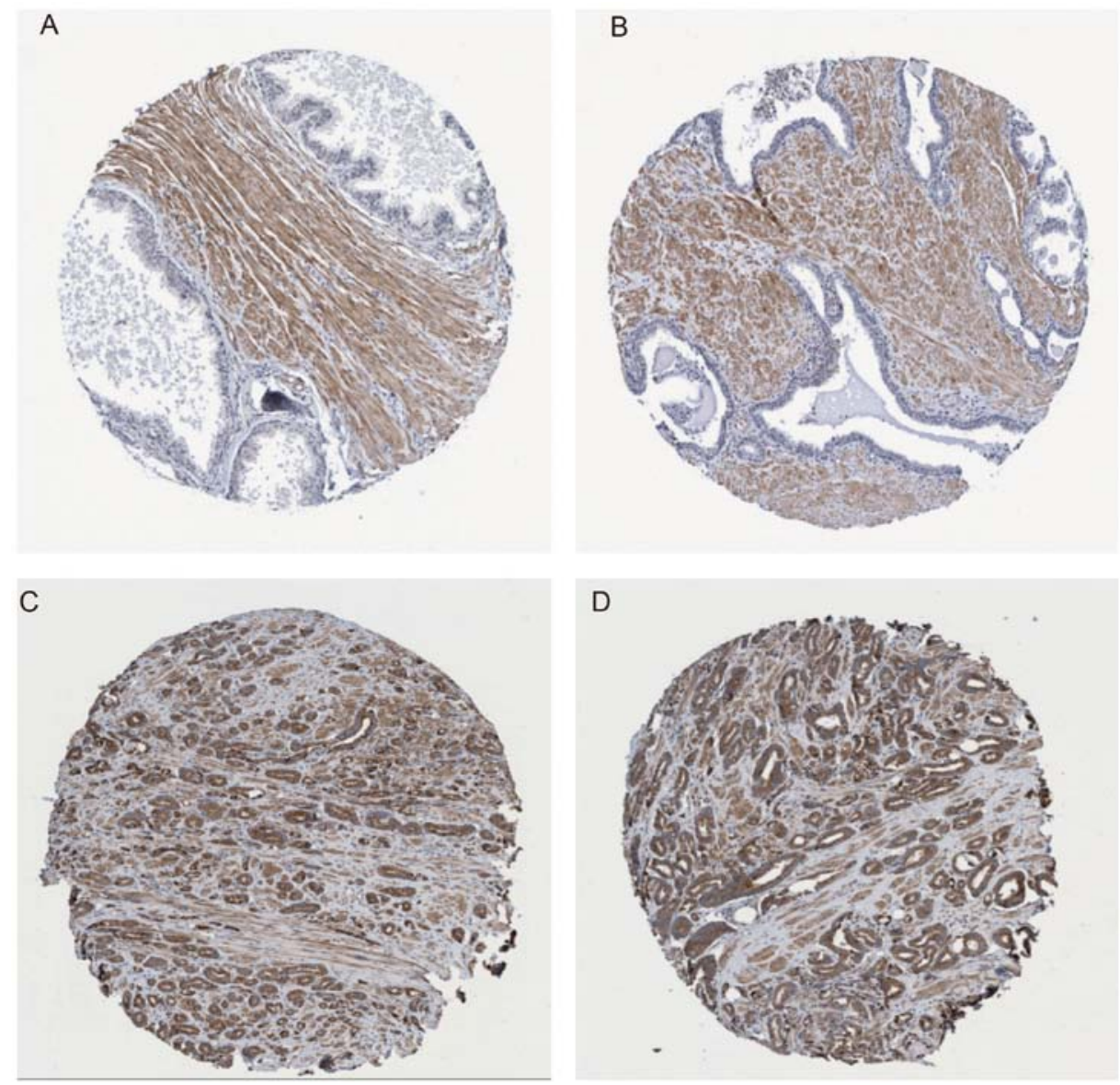

Figure 7. CALCOCO2 protein levels are increased in PCa tissue samples. Protein levels of CALCOCO2 in (A and B) normal prostate tissues (https://www.proteinatlas.org/ENSG00000136436-CALCOCO2/tissue/prostate\#img) and (C and D) PCa tissues (https://www.proteinatlas. org/ENSG00000136436-CALCOCO2/pathology/tissue/prostate+cancer\#img). CALCOCO2, calcium binding and coiled-coil domain 2; PCa, prostate cancer.

that CALCOCO2 may be associated with cancer progression (20,37,38). For example, Leymarie et al (20) observed that CALCOCO2 interacted with type I interferon production and $\mathrm{I} \kappa \mathrm{B}$ kinase/NF- $\kappa \mathrm{B}$ signaling using bioinformatics tools. Type I interferon production has been reported to be associated with antitumor immunity (37), whereas NF- $\kappa \mathrm{B}$ signaling may promote tumor cell proliferation and EMT, and suppress apoptosis of cancer cells (38). In the present study, bioinformatics analysis was performed to determine the potential mechanisms via which CALCOCO2 regulates PCa progression. A total of 190 proteins were indicated to be cofactors of CALCOCO2. A PPI network mediated by CALCOCO 2 was also constructed, which may provide useful information to understand the potential roles of CALCOCO2 in regulating $\mathrm{PCa}$ progression. Bioinformatics analysis revealed that $\mathrm{CALCOCO} 2$ was primarily associated with 'mitophagy', 'macroautophagy', 'nucleophagy', 'nucleic acid metabolic process', 'autophagosome assembly', 'regulation of transcription', 'regulation of gene expression', 'RNA metabolic process', 'zinc ion binding', 'microtubule binding' and 'transition metal ion binding'.

In conclusion, the present study demonstrated that CALCOCO2 functions as an oncogene in PCa. CALCOCO2 knockdown decreased cell proliferation and colony formation and promoted apoptosis in PCa cell lines. Bioinformatics analysis revealed that $\mathrm{CALCOCO} 2$ was involved in regulating 'autophagy', 'nucleophagy' and 'nucleic acid metabolic process' via interacting with SQSTM1. The current study also demonstrated that the CALCOCO2 protein levels were upregulated in $\mathrm{PCa}$ tissue samples. These results suggested that CALCOCO2 may be a useful diagnostic and therapeutic target in PCa.

\section{Acknowledgements}

Not applicable.

\section{Funding}

The present study was funded by the Social Development Plan of Jiangsu Province-Standardization of Key Disease Diagnosis and Treatment Projects (grant no. BE2016715) and Jiangsu Province Youth Medical Key Talent Program (grant no. QNRC2016457).

\section{Availability of data and materials}

The datasets used and/or analyzed during the current study are available from the corresponding author on reasonable request.

\section{Authors' contributions}

FC and JH were responsible for the study conception and design. FC, SW, JT and HT performed the experiments. FC, 
HT and YF analyzed and interpreted the data. All authors wrote, reviewed and revised the manuscript. All authors read and approved the final manuscript.

\section{Ethics approval and consent to participate}

Not applicable.

\section{Patient consent for publication}

Not applicable.

\section{Competing interests}

The authors declare that they have no competing interests.

\section{References}

1. Dou Z, Xu C, Donahue G, Shimi T, Pan JA, Zhu J, Ivanov A, Capell BC, Drake AM, Shah PP, et al: Autophagy mediates degradation of nuclear lamina. Nature 527: 105-109, 2015.

2. Singh R, Kaushik S, Wang Y, Xiang Y, Novak I, Komatsu M, Tanaka K, Cuervo AM and Czaja MJ: Autophagy regulates lipid metabolism. Nature 458: 1131-1135, 2009.

3. Wan X, Huang W, Yang S, Zhang Y, Pu H, Fu F, Huang Y, Wu H, $\mathrm{Li} \mathrm{T}$ and $\mathrm{Li} \mathrm{Y}$ : Identification of androgen-responsive lncRNAs as diagnostic and prognostic markers for prostate cancer. Oncotarget 7: 60503-60518, 2016

4. Zhu X, Zhou M, Liu G, Huang X, He W, Gou X and Jiang T: Autophagy activated by the c-Jun N-terminal kinase-mediated pathway protects human prostate cancer PC 3 cells from celecoxib-induced apoptosis. Exp Ther Med 13: 2348-2354, 2017.

5. Kim KY, Park KI, Kim SH, Yu SN, Park SG, Kim YW, Seo YK, Ma JY and Ahn SC: Inhibition of autophagy promotes salinomycin-induced apoptosis via reactive oxygen species-mediated $\mathrm{PI} 3 \mathrm{~K} / \mathrm{AKT} / \mathrm{mTOR}$ and ERK/p38 MAPK-dependent signaling in human prostate cancer cells. Int J Mol Sci 18: 1088, 2017.

6. Nie C, Zhou J, Qin X, Shi X, Zeng Q, Liu J, Yan S and Zhang L: Diosgenininduced autophagy and apoptosis in a human prostate cancer cell line. Mol Med Rep 14: 4349-4359, 2016.

7. Wang L, Kim D, Wise J, Shi X, Zhang Z and DiPaola RS: p62 as a therapeutic target for inhibition of autophagy in prostate cancer. Prostate 78: 390-400, 2018.

8. Chang MA, Morgado M, Warren CR, Hinton CV Farach-Carson MC and Delk NA: p62/SQSTM1 is required for cell survival of apoptosis-resistant bone metastatic prostate cancer cell lines. Prostate 74: 149-163, 2014.

9. Santanam U, Banach-Petrosky W, Abate-Shen C, Shen MM, White E and DiPaola RS: Atg7 cooperates with Pten loss to drive prostate cancer tumor growth. Genes Dev 30: 399-407, 2016.

10. Chandra P and Kumar D: Selective autophagy gets more selective: Uncoupling of autophagy flux and xenophagy flux in Mycobacterium tuberculosis-infected macrophages. Autophagy 12: 608-609, 2016.

11. Bauckman KA, Owusu-Boaitey N and Mysorekar IU: Selective autophagy: Xenophagy. Methods 75: 120-127, 2015.

12. Mao K and Klionsky DJ: Xenophagy: A battlefield between host and microbe, and a possible avenue for cancer treatment. Autophagy 13: 223-224, 2017.

13. Sui X, Liang X, Chen L, Guo C, Han W, Pan H and Li X: Bacterial xenophagy and its possible role in cancer: A potential antimicrobial strategy for cancer prevention and treatment. Autophagy 13: 237-247, 2017

14. Zhang L, Sung JJ, Yu J, Ng SC, Wong SH, Cho CH, Ng SS Chan FK and Wu WK: Xenophagy in Helicobacter pylori- and Epstein-Barr virus-induced gastric cancer. J Pathol 233: 103-112, 2014.

15. Verlhac $P$, Viret $C$ and Faure $M$ : Dual function of CALCOCO2/NDP52 during xenophagy. Autophagy 11: 965-966, 2015.

16. Verlhac P, Viret C and Faure M: Handcuffs for bacteria-NDP52 orchestrates xenophagy of intracellular Salmonella. Microb Cell 2: 214-215, 2015
17. von Muhlinen N, Akutsu M, Ravenhill BJ, Foeglein A, Bloor S, Rutherford TJ, Freund SM, Komander D and Randow F: LC3C, bound selectively by a noncanonical LIR motif in NDP52, is required for antibacterial autophagy. Mol Cell 48: 329-342, 2012.

18. Verlhac P, Gregoire IP, Azocar O, Petkova DS, Baguet J, Viret $\mathrm{C}$ and Faure M: Autophagy receptor NDP52 regulates pathogen-containing autophagosome maturation. Cell Host Microbe 17: 515-525, 2015

19. Thurston TL, Wandel MP, von Muhlinen N, Foeglein A and Randow F: Galectin 8 targets damaged vesicles for autophagy to defend cells against bacterial invasion. Nature 482: 414-418, 2012.

20. Leymarie O, Meyer L, Tafforeau L, Lotteau V, Costa BD, Delmas B, Chevalier $\mathrm{C}$ and Le Goffic R: Influenza virus protein PB1-F2 interacts with CALCOCO2 (NDP52) to modulate innate immune response. J Gen Virol 98: 1196-1208, 2017.

21. Cui F, Hu J, Fan Y, Tan J and Tang H: Knockdown of spindle pole body component 25 homolog inhibits cell proliferation and cycle progression in prostate cancer. Oncol Lett 15: 5712-5720, 2018.

22. Livak KJ and Schmittgen TD: Analysis of relative gene expression data using real-time quantitative PCR and the 2(-Delta Delta C(T)) method. Methods 25: 402-408, 2001.

23. Nabzdyk CS, Chun M, Pradhan NL, Yoshida S and LoGerfo FW: Differential susceptibility of human primary aortic and coronary artery vascular cells to RNA interference. Biochem Biophys Res Commun 425: 261-265, 2012.

24. Kanehisa M and Goto S: KEGG: Kyoto encyclopedia of genes and genomes. Nucleic Acids Res 28: 27-30, 2000.

25. Dennis GJ, Sherman BT, Hosack DA, Yang J, Gao W, Lane HC and Lempicki RA: DAVID: Database for annotation, visualization, and integrated discovery. Genome Biol 4: P3, 2003.

26. Szklarczyk D, Franceschini A, Wyder S, Forslund K, Heller D, Huerta-Cepas J, Simonovic M, Roth A, Santos A, Tsafou KP, et al: STRING v10: Protein-protein interaction networks, integrated over the tree of life. Nucleic Acids Res 43: D447-D452, 2015.

27. Shannon P, Markiel A, Ozier O, Baliga NS, Wang JT, Ramage D, Amin N, Schwikowski B and Ideker T: Cytoscape: A software environment for integrated models of biomolecular interaction networks. Genome Res 13: 2498-2504, 2003.

28. Uhlén M, Fagerberg L, Hallström BM, Lindskog C, Oksvold P, Mardinoglu A, Sivertsson A, Kampf C, Sjöstedt E, Asplund A, et al: Proteomics. Tissue-based map of the human proteome. Science 347: 1260419, 2015.

29. McFarland JM, Ho ZV, Kugener G, Dempster JM, Montgomery PG, Bryan JG, Krill-Burger JM, Green TM, Vazquez F, Boehm JS, et al: Improved estimation of cancer dependencies from large-scale RNAi screens using model-based normalization and data integration. Nat Commun 9: 4610, 2018.

30. Nakayama N, Nakayama K, Shamima Y, Ishikawa M, Katagiri A, Iida K and Miyazaki K: Gene amplification CCNE1 is related to poor survival and potential therapeutic target in ovarian cancer. Cancer 116: 2621-2634, 2010.

31. Liu Y, Zhang X, Han C, Wan G, Huang X, Ivan C, Jiang D, Rodriguez-Aguayo C, Lopez-Berestein G, Rao PH, et al: TP53 loss creates therapeutic vulnerability in colorectal cancer. Nature 520: 697-701, 2015.

32. Cancer Genome Atlas Research Network: The molecular taxonomy of primary prostate cancer. Cell 163: 1011-1025, 2015.

33. Zhong Z, Sanchez-Lopez E and Karin M: Autophagy, inflammation, and immunity: A Troika governing cancer and its treatment. Cell 166: 288-298, 2016.

34. Liu C, Xu P, Chen D, Fan X, Xu Y, Li M, Yang X and Wang C: Roles of autophagy-related genes Beclin-1 and LC3 in the development and progression of prostate cancer and benign prostatic hyperplasia. Biomed Rep 1: 855-860, 2013.

35. Jiang X, Huang Y, Liang X, Jiang F, He Y, Li T, Xu G, Zhao H Yang W, Jiang G, et al: Metastatic prostate cancer-associated P62 inhibits autophagy flux and promotes epithelial to mesenchymal transition by sustaining the level of HDAC6. Prostate 78: 426-434, 2018

36. Evan GI and Vousden KH: Proliferation, cell cycle and apoptosis in cancer. Nature 411: 342-348, 2001.

37. Fuertes MB, Woo SR, Burnett B, Fu YX and Gajewski TF: Type I interferon response and innate immune sensing of cancer. Trends Immunol 34: 67-73, 2013.

38. Xia Y, Shen S and Verma IM: NF- $\kappa$ B, an active player in human cancers. Cancer Immunol Res 2: 823-830, 2014. 